\title{
Single And Large Grain Activities At Fermilab
}

\author{
Claire Antoine \\ On behalf of Technical Division, Fermi National Accelerator Laboratory, Batavia, IL 60510 USA
}

\begin{abstract}
This paper describes the ongoing activities at Fermilab for large grains and monocrystalline niobium. In addition to acquisition of local fabrication expertise, we plan to develop an R\&D program dedicated to evidence the possible influence of crystal orientation on physical and chemical properties of niobium, such as mechanical properties, magnetic properties or surface contamination. Some considerations are also given about the morphology at grain boundaries and its role on the behavior of superconducting cavities.
\end{abstract}

Keywords: Niobium, large grain, monocrystals, grain boundaries, surface morphology, RF superconductivity PACS:

\section{INTRODUCTION}

Large grain and monocrystalline superconducting RF cavities have recently provoked a breakthrough in the SRF community. This new fabrication technique seems to bring practical as well more fundamental advantages. Slicing high RRR ingot could save us many intermediate fabrication steps like forging, rolling and recrystallization, thus reducing risk of post contamination. Hope to have to lighter processing : Buffered Chemical Etching (BCP) instead of Electropolishing (EP), reduced baking time- is also an incentive perspective. But the difficulty of forming large grain material and anisotropy in its properties must not overcome the foreseen advantages. A real breakthrough has been achieved with the recent demonstration of the feasibility of monocrystalline cavities [1]. If fabrication and supply of billet with very large central grain shows to be industrially achievable, then we can consider choosing specific orientation, depending on what parameter we want to privilege, such as forming ability, magnetic properties, work function, or transition field. Even if it appears that such cavities are not feasible on a large scale, they will provide us valuable information: their difference of behavior (or absence of difference!) compare to multigrain cavities will help us a lot to understand RF superconductivity issues.

Fermilab program aims at two complementary goals: developing local expertise on the fabrication process, so that we can rapidly switch to this new fabrication scheme if it proves valuable for ILC applications; and developing an R\&D program to support both large grain and small grain programs, and gathering complementary information on the variation of the material properties with grain orientation.

\section{VARIATION OF PROPERTIES WITH ORIENTATION}

Although body centered cubic structure has a lot of equivalent directions, there are some that correspond to dense packing of atoms (e.g. (111)), whereas some other present a looser repartition of atoms (e.g. (001)). It has consequences on the local electronic density, and affects a whole area of material properties ranging from Brillouin zones, and expectantly superconducting properties to chemical properties like diffusion coefficients. For instance, a difference of a factor 2 can be found for Young modulus depending on directions (see e.g. [2] and T. Gnäupel-Herold, this workshop), and (111) is usually considered to exhibit a better formability for BBC metals [3]. Other properties like the work function are also known to differ [4]. Oxide thickness is known to vary with orientation (see e.g. P. Russell, this workshop), and diffusion behavior upon baking is also expected to be different. In the following we will concentrate on 3 topics that we want to study more into details.

Note that if we go for specific orientation related properties, the fabrication of very large grains instead of monocrystalline cavities doesn't seem to bring any advantages. We will discuss this point latter on. 


\section{Mechanical Properties}

Mechanical properties from $\mathrm{Nb}$, in particular at low temperature are not well known, only a few studies of very pure $\mathrm{Nb}$ can be found in literature [see e.g. 5, 6]. Actual data at room temperature should help us to predict forming behavior. Cold data are mandatory to predict the mechanical resistance of the formed cavity, how it react to pressure changes, vibrations, and any other stresses encountering during cool down and RF operation. We know for instance that welding decrease a lot the mechanical resistance of the material [3], but we don't know how it evolves when the material is cold (liquid helium). Recent problem occurred at Fermilab with brittle transition found on a coupler show how critical this issue is [7]. Neither do we know now how a few large grains material will evolve at cold temperature: is there for instance any "dangerous" orientation or misorientations between grains?

We intend implement our tensile test facility with cold operation and start a study of the evolution of mechanical properties of $\mathrm{Nb}$ upon various conditions, in particular recrystallization/recovering and welding.

\section{Magnetic Properties}

As we quoted before, superconducting properties are also expected to vary with orientation, although it is difficult to know in what extent for the actual $\mathrm{Nb}$ we are using. In particular we can measure surface superconducting properties, mainly $\mathrm{H}_{\mathrm{C} 3}$, with the help of a magnetometer. Such technique has been applied to polycrystalline $\mathrm{Nb}$ samples [8] and proved to be very sensitive to surface treatments and baking. If we apply this technique to monocrystalline samples and show e.g. that some crystal orientation exhibit a higher $\mathrm{B}_{\mathrm{C} 3}$, it might become a strong criteria for the selection of a proper orientation. It will also give interesting clues about the origin of non uniform behavior in polycrystalline material.

\section{Chemical Properties}

Crystalline orientation is known to influence oxidation as well as dissolution, since the mobility of the atoms depends on their crystalline location: for instance, (111) planes are the closest packed, but their atoms also exhibit the highest lateral mobility. Chemical etching, as well as electropolishing of surfaces (not to mention any wet processing, like e.g. rinsing), proceed through an oxidation reaction. During oxidation it is well known that injection of interstitial atoms compete with the formation of the oxide layer (see e.g. [9]). Once again we are expecting a different behavior for a dense direction like (111) compare to a loose packed direction like e.g. (001). Indeed, P. Russell (this workshop) has shown that oxide thickness on $\mathrm{Nb}(111)$ is $\sim 7.5 \mathrm{~nm}$ while on $\mathrm{Nb}(100)$ it is $\sim 4.9 \mathrm{~nm}$. The next important information we need is to determine wherever the oxide thickness is anti-correlated with interstitial oxygen distribution inside the superconductor like it could be intuitively guessed. Indeed, the TEM pictures show that the interface between the oxide layer and the $\mathrm{Nb}$ matrix is not clearly delimited. It is very difficult to tell if it is the serration model proposed by Halbritter [10], sub-oxides or interstitial oxygen atoms ${ }^{1}$. But the differences of distribution of oxygen atoms in this region are expected to affect superconductivity, in particular the superconducting gap and the mean free path. The existence of metallic sub-oxides is also expected to affect the surface superconductivity through proximity effect.

Moreover the roughness observed after chemical etching is due to the different orientation found in polycrystalline material, and their different etching rates.

\section{Orientation and Surface Roughness}

One of the main advantages from monocrystalline cavities is that their surface can be processed with chemical buffered polishing (BCP) rather than electropolishing (EP), BCP being somewhat easier to apply and more reproducible in results. Usually BCP produces surface 5 to 10 times rougher than EP. Apparently, than in the case of large grain the roughness after BCP is reduced. In the following chapter,

${ }^{1}$ Recently it was shown by X-ray diffraction that on atmospherically oxidized $\mathrm{Nb}$ exhibit only one monolayer of NbO, and that it tends to grow upon baking [. Deleussy, MPI-Stuttgart, personal communication] This confirms results inferred trough XPS deconvolution. 
we will discuss about the origin of the roughness of BCP surfaces and how it can affect superconductivity.

\section{Roughness Measurement}

One of the main problems in comparing roughness data arise from the fact that roughness depends on observation scale. Hence most of the data found in literature usually can not be compared. Only measures made in the same conditions can be used. An alternative to this problem is to use fractal approach. Indeed, most polished crystal surfaces display a fractal behavior, that is, the root-mean-squared roughness height varies as $\sigma \approx \mathrm{bx}^{\alpha}$, where $\mathrm{b}$ is a constant and $\alpha$ the roughness exponent. This approach applied to Kapitza resistance gave interesting results ([11] and references therein). Nevertheless, roughness doesn't fully characterize the influence of morphology on cavity behavior. Indeed the 2 schematic surfaces from figure 1 exhibit exactly the same roughness, while their behavior in an electromagnetic field would be very different.
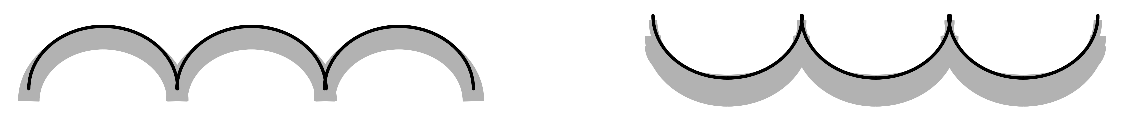

FIGURE 1. Schematic surfaces with similar roughness but very different behavior in EM field.

A topological approach allows better characterizing the surface for our purpose: equivalent conformal ellipsis. This technique is described in detail elsewhere [12]. It consist to details the analyzed surface into micro-pyramids, and to add up the projection of each micro-facets in each directions of the space. The data are transformed using a conformal mapping that retains only the surface orientations. The resulting spatial angularity of the micro-facets is implemented in a parameterized representation, an ellipsoid. The resulting ellipsoid is equivalent to the initial surface from a topological point of view and has the same developed surface. This approach can be used either to define a mean surface value or even model a single step. The field enhancement factor of such an ellipsoid can be easily calculated $[13,14,16]$.
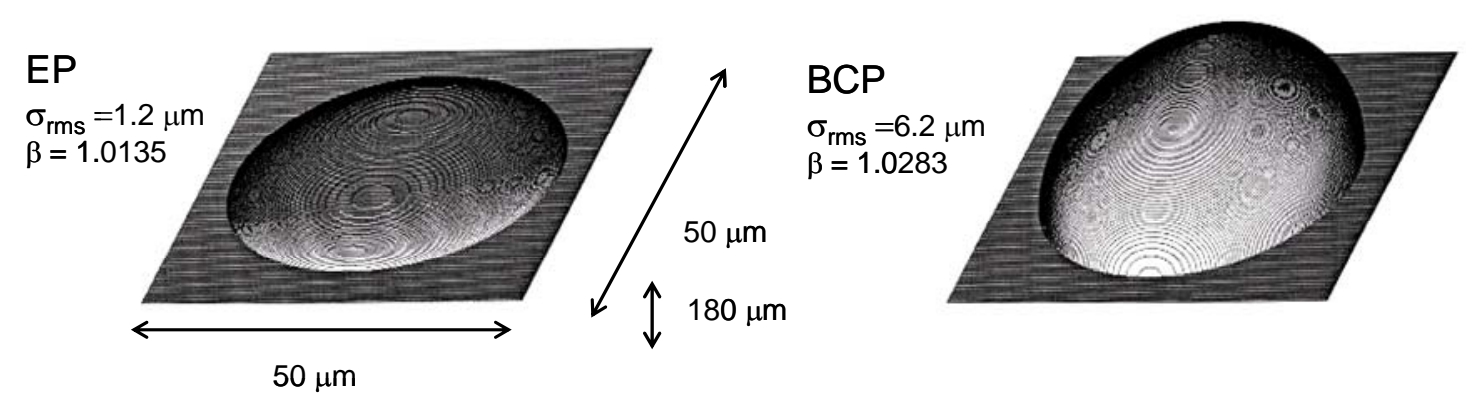

FIGURE 2. Equivalent conformal ellipsoids for EP and BCP polycrystalline surfaces, along with the measured roughness $(\sigma)$ and the field enhancement factor $(\beta$, mean value). $\sim 10$ grains are scanned.

Figure 2 shows that although the roughness a well as the shape factor (vertical axis of the ellipsoid) seems to differ a lot between a chemically polished surface and an electropolished one. Is this example, 10 grains were scanned, which explains why the roughness values are quite high compare to usual values published previously ; indeed if the scanned surface is too small the actual roughness due to grain boundaries cannot be detected. Nevertheless, there is not significant difference in the mean value of the field enhancement factor, showing that the morphology influence is not a global one. Moreover it was shown that in the heat affected area, near the welding seam, roughness and shape factor are about ten fold higher [13], and field enhancement factor is not negligible any more [15]. As we will discuss later, we think that the steps that appear at the grain boundaries near the welding seam are the main cause for early quenches in BCP treated cavities. Thus the use of monocrystals would certainly allow using this surface treatment without being penalized. On the other hand, the use of large grain might not bring any advantages except if we can prevent recrystallization at the weld, and if we get grains with close orientation that do not exhibit differentiated etching rates. 


\section{Surface Roughness Origin}

In the case of $\mathrm{BCP}$, roughness tends to increases with time since the etching rate depends on the superficial energy and of the mobility of atoms on a particular face. Thus we do not only observe different etching rate with the crystals orientation, but also preferential etching at obvious crystalline defects like grain boundaries or dislocation emergences at the surface (it is the origin of the "etching figures" that we observe on the grain surface). Roughness is observed mainly on the micron scale, at very low scale the both processing techniques exhibit the same kind of surface features (see figure 3 ).
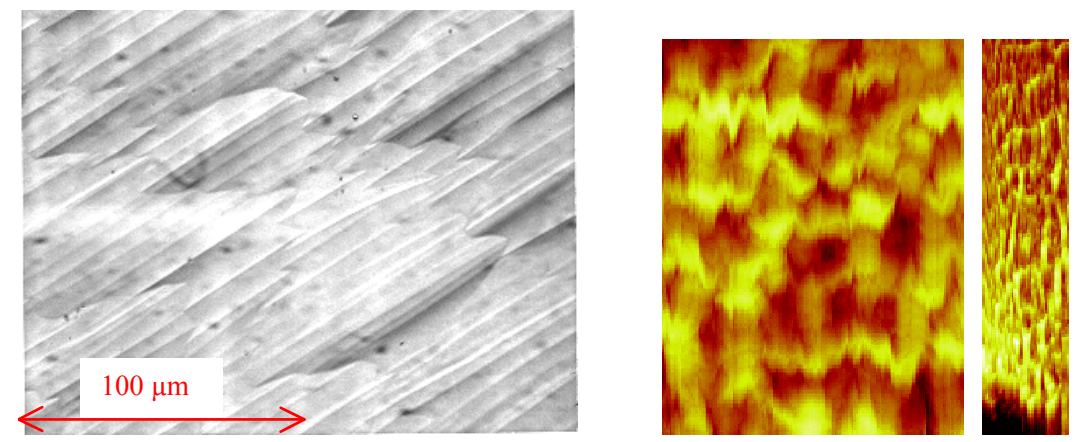

\section{BCP}
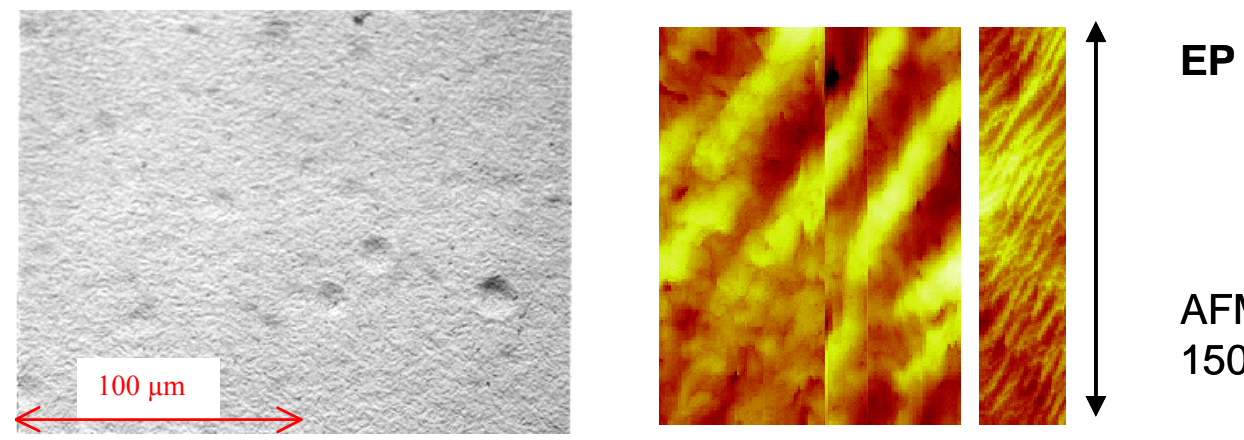

AFM

$1500 \mathrm{~nm}$

FIGURE 3. Surface aspect of BCP and EP on a (110) monocrystals at different scales (left: optical, right, tunneling microscopy). At very low scale, the roughness is alike with both surface treatments, whereas at the micron scale, we can clearly observe faceting due to the preferential etching with BCP

\section{Grain Boundaries And Superconductivity}

Two hypotheses might be proposed about the effect of grain boundaries: either they exhibit a depleted superconductivity du to e.g. disorder of the lattice, or impurities segregation, or it can be a morphologic effect due to surface roughness. They are indeed some indications for both contributions. Evidences of referential flux penetration at grain boundaries have been shown in this conference, along with some magneto-optic and TEM results (see Peter Lee's paper). Similar measurement made on a monocrystals prepared with an artificial notch exhibited field enhancement contrast, in particular when the magnetic field is parallel to the surface and perpendicular to the notch, showing that morphological effect cannot be neglected. Such a configuration is very alike to what can be found in cavities near the welding seam.

Recently it was proposed that field enhancement on the edge of the grain boundaries can trigger locally an early quench in the case of BCP cavities [13]. Indeed, in the case of welded cavity, we have very large grains and steep edges in the thermal affected zone near the weld. This area is not large enough to explain the hot spots as well as the Q drop observed on the whole surface of the cavities, but once close to the transition field, only one grain edge with local increased field is enough to get a localized transition.

\section{DISCUSSION AND CONCLUSION}

It is now clear that monocrystalline cavities can be satisfactorily prepared with a BCP and achieve very high performances. Nevertheless, the obtaining of very large grains is so difficult that we might not gain significant cost reduction. Large grains are expected to have the same problems as small grains, since some recrystallization appears, especially at triple points. Their forming behavior is not very good due to the large difference of behavior of each grain, and higher failure rate is expected. Usually a mechanical 
polishing is needed after forming and then again cost reduction might not be significant. If we could prepare large grain material with grains with very close orientations, we could overcome such difficulties, since the difference of behavior (forming, etching) might be not too high. Nevertheless, large grain and monocrystalline cavities offer an important tool to understand high field RF superconductivity, freeing us from a part of the natural variability of the material over large surfaces.

\section{REFERENCES}

1. W. Singer et al, this conference

2. Pal-Val, P.P., V.D. Natsik, and L.N. Pal-Val, "Dynamic elastic moduli of Niobium at low temperature: their dependance in the Normal state, the influence of the superconducting transition, and dislocation effects", Low Temperature Physics, 32(2): p. 169-185, (2006).

3. Jiang,. H. et al, "Evolution of recrystallization textures from cold rolling textures in high purity Nb", PAC 2003, (2003).

4. J. Norem, http://ilc-

dms.fnal.gov/Workgroups/SRFMaterials/SRFMaterialMeetings/MidwestSRFMatMeetNov06/Presentations/Norem_Pulsed_Power Processing.pdf

5. J.F. Fries, "Influence des elements interstitiels $(\mathrm{O}, \mathrm{C}, \mathrm{N})$ sur le comportement plastique en traction du niobium polycristallin entre -253 C et 850 C", PhD Thesis, Orsay, France, (1972)

6. Rao, M. G., and Kneisel, P., "Mechanical Properties of High RRR Niobium at Cryogenic Temperatures", Advances in Cryogenic Engineering Vol., Plenum Press, New York, pp. 1383-1390 (1994) and G. R. Mynenil and H. Umezawa, "Variation of Mechanical Properties of High RRR And Reactor Grade Niobium With Heat Treatments", UHPM 2003, Saint Etienne, France June 16-20, (2003).

7. W.D. Moeller, TTC meeting, Kek, (2006), https://indico.desy.de/getFile.py/access?contribId=70\&amp;sessionId=9\&amp;resId=0\&amp;materialId=slides\&amp;confId=92

8. Casalbuoni, S., et al., "Surface superconductivity in $\mathrm{Nb}$ for superconducting RF cavities", Nuclear Instrumentation and Methods in Physical Research A, 538: p. 45-64, (2005).

9. Hellwig, "Oxidation of epitaxial $\mathrm{Nb}$ films: Oxygen dissolution and oxide formation", PhD, Bochum, Germany, (2000)

10. J. Halbritter, "On the oxidation and on the superconductivity of niobium",. Applied Physics A, 43: pp. 1-28, (1987).

11. Amrit, "Nanoscale heat conduction at a silicon-superfluid helium boundary", Superlattices and Microstructures,. 35: p. 187-194, (2004).

12. C. Roques-Carmes, J.F. Quiniou, N. Bodin, G. Monteil, "Geometrical Description of Surface Topography by Means of an Equivalent Conformal Profile Model", 7th Int, Conf, on Metrology and Properties of Engineering Surfaces 175-181 (1997)

13. S. Berry, C. Antoine, and M. Desmons. "Surface morphology at the quench site", EPAC 2004, Lucern, Switzerland, (2004) and S. Berry, et al. "Topologic analysis of samples and cavities: a new tool for morphologic inspection of quench site", 11th workshop on RF Superconductivity, Lübeck, Germany, (2003)

14. P. Bauer, Personal communication

15. J. Knobloch, et al., "High-Field Q Slope in superconducting cavities du to magnetic field enhancement at grain boundaries", 9th Workshop on RF Superconductivity,. Santa Fe, NM, USA, (1999).

16. J. A. Osborn, “ demagnetizing factors in the general ellipsopid”, Phys. Rev., 67 (1-2), pp 347-351, (1945) 